\title{
Development and Testing of an Instrument to Measure Contextual Factors Influencing Self-Care Decisions Among Adults with Chronic Illness
}

\section{Shayleigh Dickson Page ( $\nabla$ dicksons@nursing.upenn.edu )}

University of Pennsylvania School of Nursing https://orcid.org/0000-0002-5416-5818

Christopher Lee

Boston College - William F Connell School of Nursing

\section{Subhash Aryal}

University of Pennsylvania School of Nursing

\section{Kenneth Freedland}

Washington University School of Medicine in Saint Louis: Washington University in St Louis School of Medicine

\section{Anna Stromberg}

Linköping University: Linkopings universitet

\section{Ercole Vellone}

University of Rome Tor Vergata: Universita degli Studi di Roma Tor Vergata

\section{Heleen Westland}

University Medical Center Utrecht

\section{Douglas J Wiebe}

University of Pennsylvania Perelman School of Medicine

\section{Tiny Jaarsma}

Linköping University: Linkopings universitet

\section{Barbara Riegel}

University of Pennsylvania School of Nursing

\section{Research}

Keywords: Self-Care, Decision Making, Chronic Illness, Instrument Development, Psychometrics

Posted Date: September 14th, 2021

DOI: https://doi.org/10.21203/rs.3.rs-869590/v1

License: (c) (1) This work is licensed under a Creative Commons Attribution 4.0 International License. Read Full License 


\section{Abstract}

Background: Adults with chronic illness frequently experience bothersome symptoms (e.g., pain). Decisions about how to manage these symptoms are complex and influenced by factors related to the patient, their illness, and their environment. The naturalistic decision-making framework describes decision-making when conditions are dynamically evolving, and the decision maker is uncertain because the situation is ambiguous and missing information. The contextual factors influencing decisions include time stress, the perception of high stakes, and input from other individuals, which may facilitate or complicate the decision about the self-care of symptoms. There is no valid instrument to measure these contextual factors. The purpose of this study was to develop and test a self-report instrument measuring the contextual factors that influence self-care decisions about symptoms.

Methods: Items were drafted from the literature and refined with patient input. Content validity of the instrument was evaluated using a Delphi survey of expert clinicians and researchers, and cognitive interviews with adults with chronic illness. Psychometric testing included exploratory factor analysis to test dimensionality, item response theory-based approaches for item recalibration, confirmatory factor analysis to generate factor determinacy scores, and evaluation of construct validity.

Results: The content validity of the Self-Care Decisions Scale is excellent with all items achieving a content validity index of greater than 0.78 in the Delphi survey of experts $(n=12)$. Adults with chronic illness $(n=5)$ endorsed the relevance, comprehensiveness, and comprehensibility of the instrument during cognitive interviews. Initial psychometric testing $(n=431)$ revealed a 6 -factor multidimensional structure that was further refined for precision, and high multidimensional reliability. In construct validity testing, there were modest associations with some scales of the Melbourne Decision Making Questionnaire and the Self-Care of Chronic Illness Inventory.

Conclusion: The Self-Care Decisions Scale is a 27-item self-report instrument that measures the extent to which contextual factors influence decisions about symptoms of chronic illness with six scales reflecting naturalistic decision making (external, urgency, uncertainty, cognitive/affective, waiting/cue competition, and concealment). The scale can support research that aims to better understand how adults with chronic illness make decisions in response to symptoms. Additional testing of the instrument is needed to evaluate clinical utility.

\section{Background}

Adults with chronic illness often experience symptoms that interfere with daily life. For example, shortness of breath may limit the distance someone with asthma can walk without taking a break. Selfcare of chronic illness includes evaluating changes in physical and emotional signs and symptoms, determining if action is needed, and deciding which action to take (1). Self-care management involves the implementation and evaluation of the effectiveness of the chosen action (e.g., use inhaler for shortness of breath). 
How adults with chronic illness make decisions about what to do when experiencing symptoms is poorly understood. The naturalistic decision making framework may help to explain how such decisions are made. Naturalistic decision making focuses on how people use experience to make decisions and how contextual factors influence this process (2). The decision maker may experience uncertainty when the situation is ambiguous, the environment is changing, or necessary information is missing. For example, a symptom may be new, or an individual may be unsure what caused the symptom. Decisions may also be influenced by time stress (e.g., symptom changes quickly), the perception that there is much at stake (e.g., symptom is severe), and conflicting input from multiple individuals (2).

Previous work suggests that self-care decisions made by adults fit within the naturalistic decision making framework. In a qualitative analysis, Riegel, Dickson (3) found that the decisions made by adults with chronic heart failure were influenced by experience, decision characteristics (e.g., uncertainty, ambiguity, high stakes, urgency, illness characteristics, and involvement of others in the decision making process), and personal goals. Further, situation awareness (i.e., recognition and interpretation of the symptom) and mental simulation (i.e., mentally thinking through options for "what to do") were integral to the decision-making process.

In spite of evidence that these contextual factors influence the self-care decisions made by patients about symptoms, there are no valid instruments to measure these factors. Measurement of contextual factors influencing self-care decisions about symptoms is important for advancing research in self-care and improving the clinical care of adults with chronic illness. If investigators can identify factors that influence self-care decisions, they can design tailored interventions to address specific barriers. The aims of this study were to (i) develop a theoretically based and clinically relevant self-report instrument that measures contextual factors influencing self-care decisions about symptoms, and (ii) test its psychometric properties, including dimensionality, construct validity, precision, and reliability.

\section{Methods}

This study was conducted in two phases: (i) instrument development and (ii) formal psychometric testing (Figure 1).

Figure 1: Instrument Development and Formal Psychometric Testing Process.

The COnsensus-based Standards for the selection of health Measurement INstruments (COSMIN; www.cosmin.nl) guided the instrument development and content validity testing, and item response theory guided the initial psychometric testing. Institutional Review Board approval for this study was obtained from the University of Pennsylvania.

\section{Instrument Development}

Step 1: item generation 
First, we identified 10 contextual factors from the literature that are thought to influence self-care decisions: prior experience, competing personal goals, uncertainty and ambiguity, urgency, situation awareness, involvement of multiple individuals, interpretation of symptom meaning, illness characteristics, dynamically evolving conditions, and high stakes. Next, we generated a preliminary list of items describing how these factors influence the response to bothersome symptoms based on the foundational work on naturalistic decision making (2) as well as the application of naturalistic decision making to self-care decisions in adults with heart failure (3). The authors discussed and revised the items as well as the instrument instructions and scoring format until consensus was reached on an initial instrument draft.

\section{Step 2: item refinement with patient input}

We then conducted cognitive interviews with adults with chronic illness. The purpose of these interviews was three-fold: 1) to assess the relevance of the proposed items to the experience of having a chronic illness, 2) to ensure that patients understood the items, and 3) to improve the comprehensiveness of the instrument by asking if any items were missing. Adults with at least one of five chronic illnesses (arthritis, asthma, chronic obstructive pulmonary disease, diabetes mellitus, and/or heart failure) were recruited through Researchmatch.org, a website sponsored by the National Institutes of Health on which people from the United States can volunteer to participate in research. These conditions were selected because they are common and often symptomatic. Eligibility criteria included age $>18$ years and currently experiencing at least one symptom of a chronic illness. Interviews were completed by the first author either by phone or video conference. SP, BR, TJ, AS, HW, and EV discussed the results of the cognitive interviews and reached consensus on changes to items.

\section{Step 3: content validity testing}

Content validity is the degree to which the content of the instrument reflects the construct (i.e., naturalistic decision making) that the instrument was designed to measure (4). The COSMIN methodology for evaluating content validity defines three properties of content validity (relevance, comprehensiveness, and comprehensibility) and further recommends that both patients and professionals are involved in the validation process (5). Thus, we evaluated the content validity of the instrument in two ways: (i) a Delphi survey of clinicians and researchers and (ii) cognitive interviews with adults with chronic illness.

\section{Step 3a: Delphi survey}

The Delphi technique uses structured questionnaires that are distributed in iterative rounds to a group of experts who remain anonymous to each other throughout the process (6). For the Delphi survey, we defined experts as (i) clinicians who routinely help adults make decisions about their chronic illnesses and (ii) researchers who have published on decision making related to chronic illness in the scientific literature. Experts were identified through a Facebook discussion on the topic of decision-making in selfcare, a literature search on decision-making in chronic illness, and the professional networks of the study authors. The Delphi survey was completed electronically using Qualtrics (Provo, UT). Respondents rated 
the relevance of items to the construct of naturalistic decision making on a 4-point scale (not relevant, somewhat relevant, quite relevant, highly relevant). The comprehensibility of items was rated dichotomously (clear, not clear). Respondents had the opportunity to suggest new items to support comprehensiveness of the instrument and ensure that no facets of the construct were omitted. Finally, respondents provided feedback on the clarity of the proposed instrument instructions and the scoring format.

After each round, the Content Validity Index (CVI) of each item (I-CVI) was calculated by dividing the number of respondents reporting that an item was "quite relevant" or "highly relevant" by the total number of respondents (7). An I-CVI greater than 0.78 is considered evidence of good content validity(7). Thus, to be retained without revision, the I-CVI had to be 0.78 or higher. Consensus on clarity was defined as at least $75 \%$ of the respondents agreeing that the item was clear. SP, BR, TJ, AS, HW, and EV met to discuss responses following each round of the Delphi survey. Items were retained, revised, or deleted following discussion of the I-CVI and clarity data as well as the respondents' open-ended suggestions.

The Content Validity of the Scale (S-CVI) was calculated at the conclusion of the Delphi survey. We report the average of the I-CVIs for all items on the scale (i.e., S-CVI/Ave). According to Polit, Beck (7), a S$\mathrm{CVI} /$ Ave greater than 0.90 indicates excellent content validity.

\section{Step 3b: Cognitive Interviews}

Following the Delphi survey, cognitive interviews with a second set of adults with chronic illness were completed to ensure that the revised items remained relevant to their experience and to assess comprehensiveness and comprehensibility of the instrument. Participants were again recruited through Researchmatch.org using the same inclusion criteria previously described. Participants were read the instrument instructions followed by each item. Per the instrument instruction, they rated how much the item influenced their decision on a 5-point Likert Scale from "not at all" to "a great deal". Participants were encouraged to "think aloud" and describe how they arrived at each answer. They also provided feedback on the clarity of the instrument instructions and Likert scale. To elicit more information, three types of verbal probing techniques were used: 1) comprehensiveness/ interpretation probes (e.g., why do you think...?), 2) paraphrasing (e.g., please repeat that statement in your own words), 3) general probes (e.g., how did you arrive at that answer?) (8).

\section{Formal Psychometric Testing}

\section{Sample}

Participants were recruited through Reaserchmatch.org for psychometric testing of the newly developed Self- Care Decisions Scale. Invitations to participate were sent to adults (age $>18 y$ ) with at least one chronic condition. Chronic condition was defined as any of the symptomatic physical or mental health conditions that are included on the list of chronic conditions published by the Office of the Assistant Secretary for Health in the Department of Health and Human Services of the United States (9). Additional 
eligibility criteria included currently experiencing at least one symptom of the chronic illness. Surveys were completed electronically using Qualtrics (Provo, UT).

\section{Step 4: Dimensionality \& Recalibration}

Descriptive statistics of central tendency and dispersion were used to describe the sample. Exploratory factor analysis was used to test dimensionality; response options were handled as ordered categorical data, and weighted least squares mean and variance adjustment and geomin oblique rotation (with a primary loading cutoff $>0.40$, and loading $<0.30$ on alternative factors) were used (10). Models ranging from 1 to 8 factors were compared using cutoff values of model fit (i.e., root mean square error of approximation (RMSEA) $<0.05$, comparative fit index (CFI) and Tucker-Lewis index (TLI) of $\geq 0.95$, and standardized root mean square residual $($ SRMR $)<0.08)(11,12)$.

Graded response item response theory (IRT)-based approaches were used within each factor for recalibration using information on item discrimination (slope and significance), item difficulty (boundary and category characteristic curves), as well as item and test information (item and test information curves)(13).

\section{Step 5: Construct Validity}

No measure of the contextual factors influencing decision making as described in the naturalistic decision making framework exists, so we chose to assess convergent validity, the degree to which the new measure is related to other measures of decision-making. We compared each recalibrated Self-Care Decisions Scale with the Melbourne Decision-Making questionnaire (Melbourne DMQ) domains. The Melbourne DMQ measures four patterns for coping with decisional conflict: vigilance, hypervigilance, buck passing and procrastination (14). The coping pattern of vigilance involves clarifying objectives, canvassing an array of alternatives, searching for relevant information, assimilating that information, and evaluating alternatives before making a choice. The pattern of hypervigilance involves frantic searching, time pressure, and impulsive choice of a contrived solution. Buck passing is described as an avoidance style associated with defensiveness and dependency. Finally, procrastination is another form of defensive avoidance that involves delaying decision making. Higher scores indicate a preference for that coping pattern and vigilance is negatively correlated with the other patterns. The scale alpha coefficient reliabilities ranged from 0.74 to 0.87 in a sample of 2018 participants from six countries (14). We hypothesized that each recalibrated scale on the Self-Care Decisions Scale would be significantly associated with Melbourne DMQ domains. Linear correlations with Bonferroni correction were computed to test these hypotheses.

Criterion validity is the extent to which one measure predicts scores on another measure. To evaluate criterion validity, we assessed the degree to which scores on the Self-Care Decisions Scale predict adequate self-care, using the Self-Care of Chronic Illness Inventory (SC-CII), a 20-item self-report generic measure of self-care based on the Theory of Self-Care of Chronic Illness (15). The SC-Cll includes three scales: Self-Care Maintenance, Self-Care Monitoring, and Self-Care Management. Scores range from 0- 
100 and higher scores indicate better self-care. A cut-point of $\geq 70$ is used to indicate self-care adequacy on each scale (16). The Self-Care Management scale is multidimensional, thus reliability is calculated using the global reliability index (17). Reliability of this scale was 0.71 in a sample of 400 adults with chronic illness (15). We hypothesized that adequate self-care management would be positively associated with the 'Urgency' scale in the Self-Care Decisions Scale and negatively associated with the Self-Care Decisions Scale 'Uncertainty' scale, discussed further below. Two-sample t-tests were used to compare Self-Care Decisions Scale scores between groups of individuals with adequate and inadequate self-care management. Hedge's $g$ is reported for effect size.

\section{Step 6: Precision \& Reliability}

IRT test information function curves were generated to display the range of each construct where recalibrated scales of the Self-Care Decisions Scale are most accurate. Multidimensional reliability was quantified using factor determinacy scores for the recalibrated Self-Care Decisions Scale in confirmatory factor analysis.

Factor analyses were performed in Mplus v8 (Los Angeles, CA), and IRT models and validity testing were performed in Stata v16 (College Station, TX). Full information maximum likelihood estimation (FIML) was used to impute the $0.3 \%$ of data that were missing at random.

\section{Results}

\section{Instrument Development}

\section{Step 1: item generation}

The instrument instructions directed survey respondents to think about the last time that they had a bothersome symptom of their chronic illness and then rate how much each item influenced their decision about what to do in response to that symptom. The initial draft of the instrument included 42 items, with several items for each of the 10 contextual factors. For example, for prior experience, items captured both having experience (e.g., I thought about similar past decisions) and lack of experience (e.g., the symptom was new to me). Each item was rated on a 5-point Likert scale with response options of not at all (1), a little (2), some (3), a lot (4), and a great deal (5).

\section{Step 2: item refinement with patient input}

Five adult women, ages 43-71, completed the cognitive interviews. Each had multiple chronic conditions and had been living with at least one symptomatic chronic condition for more than 10 years. Based on the responses of these adults, 23 items were retained as written, 7 items were revised, and 11 items were added. Item revisions were made to improve clarity. For example, "I recognized this from last time" was changed to "I recognized this symptom from last time". Items were added when participants identified that a factor that influences their decision was not captured by existing items. For example, a participant identified that her decision making is affected by depressive symptoms, so the item "I felt too down, so I 
put off making a decision" was added. Finally, 12 items were deleted as irrelevant (8 items) or redundant (4 items). The refined draft of the instrument included 41 items.

\section{Step 3a: Delphi survey}

Twenty-six experts were invited via email to complete the Delphi survey. There were 12 respondents ( 9 female, 3 male) in round 1 and all 12 respondents also completed round 2. Experts were from United States $(n=7)$, Italy $(n=4)$, and Germany $(n=1)$ and reported an average of 13 years of experience caring for adults with chronic illness. Eleven out of 12 experts had a PhD and one had a master's degree. I-CVI and clarity data for each Delphi round are summarized in Table 1.

\section{Table 1. I-CVI and Clarity Data by Delphi Round}

\begin{tabular}{|c|c|c|c|c|c|c|c|}
\hline & $\begin{array}{l}\text { \# of } \\
\text { experts }\end{array}$ & $\begin{array}{l}\text { \# of } \\
\text { items in } \\
\text { round }\end{array}$ & $\begin{array}{l}\mathrm{I}-\left.\mathrm{CV}\right|^{\mathrm{a}} \\
\text { range }\end{array}$ & $\begin{array}{l}\% \text { of }{ }^{-} \\
\mathrm{CVI}> \\
0.78^{\mathrm{b}}\end{array}$ & $\begin{array}{l}\text { Clarity }{ }^{c} \\
\text { range }\end{array}$ & $\begin{array}{l}\text { Items rated as clear by } \\
\leq 75 \% \text { of experts }\end{array}$ & $\begin{array}{l}\text { Items } \\
\text { deleted } \\
\text { or added } \\
\text { in round }\end{array}$ \\
\hline $\begin{array}{l}\text { Round } \\
1\end{array}$ & 12 & 41 & $\begin{array}{l}0.5- \\
1.0\end{array}$ & $95 \%$ & $\begin{array}{l}42- \\
100 \%\end{array}$ & 12 items & $\begin{array}{l}9 \text { deletec } \\
5 \text { added }\end{array}$ \\
\hline $\begin{array}{l}\text { Round } \\
2\end{array}$ & 12 & 37 & $\begin{array}{l}0.83- \\
1.0\end{array}$ & $100 \%$ & $\begin{array}{l}75- \\
100 \%\end{array}$ & 0 items & 2 added \\
\hline
\end{tabular}

a Item Content Validity Index $(\mathrm{I}-\mathrm{CVI})=$ number of respondents who rated the item as 'highly relevant' or 'quite relevant' divided by total number of respondents.

${ }^{\mathrm{b}} \mathrm{An} \mathrm{I-CVI}$ of 0.78 or higher indicates good content validity at the item level

${ }^{\mathrm{C}}$ Clarity $=$ number of respondents who rated the item as clear divided by the total number of respondents

${ }^{d}$ Items rated as clear by less than $75 \%$ of experts required revision

The Delphi survey was closed after the second round as consensus on item relevance and clarity was achieved. The S-CVI/Ave of this 39-item instrument was excellent at 0.92 .

Step 3b: cognitive interviews

Five adults ( 3 female, 2 male), ages 44-70, completed the second round of cognitive interviews. Four adults had multiple chronic conditions, while one adult had only one condition. Two adults had been diagnosed in the last 3 years, while 3 adults reported having at least one symptomatic chronic condition for more than 10 years. 
In these cognitive interviews, respondents reported that items were relevant to their experience and the instrument was comprehensive. No new items were suggested. For three items, participants reported confusion about wording and endorsed multiple interpretations of the item. These three items were deleted because there were other items that captured the same contextual factor and were clearer to participants. One item, "I worried about the cost of treatment", was deleted because it reflected access to insurance coverage, which differs across countries. The instrument instructions were also shortened and simplified based on participant feedback. The anchors of the 5-point scale were change to "No Influence" (1) and "A Lot of Influence" (5). Following content validity testing, the instrument contained 35 items.

\section{Psychometric Testing}

Invitations to participate were sent to 1,127 individuals who expressed interested in the study on Researchmatch.org. A total of 431 individuals completed the survey for a response rate of $38.2 \%$. The typical participant was female, White, non-Hispanic, with at least some college education (Table 2).

\section{Table 2: Participant Characteristics $(n=431)$}




\begin{tabular}{|c|c|}
\hline & $\mathrm{n}(\%)$ \\
\hline mean (sd) & $54.93(16.15)$ \\
\hline \multicolumn{2}{|l|}{ Gender $(n=426)$} \\
\hline Female & $302(70.1)$ \\
\hline \multicolumn{2}{|l|}{ Race $(n=425)$} \\
\hline White & $375(87)$ \\
\hline Black & $20(4.6)$ \\
\hline Native American/Alaska Native & $2(0.5)$ \\
\hline Asian & $6(1.4)$ \\
\hline Native Hawaiian/Pacific Islander & $1(0.2)$ \\
\hline Mixed (two or more) & $21(4.9)$ \\
\hline \multicolumn{2}{|l|}{ Ethnicity $(n=427)$} \\
\hline Hispanic & $19(4.4)$ \\
\hline \multicolumn{2}{|l|}{ Education $(n=430)$} \\
\hline High School or Less & $23(5.3)$ \\
\hline Some College & $74(17.2)$ \\
\hline Associate's or Bachelor's Degree & $179(41.5)$ \\
\hline Master's Degree & $102(23.7)$ \\
\hline Professional or Doctoral Degree & $45(10.4)$ \\
\hline Other & $7(1.6)$ \\
\hline
\end{tabular}

\section{Employment $(\mathrm{n}=430)$}

Full Time

$122(28.3)$

Part Time

$41(9.5)$

Unemployed

$19(4.4)$

Unable to work due to illness/disability

$97(22.5)$

Retired

$134(31.1)$

Other

$17(3.9)$

Finances $(n=420)$

Have enough or more than enough to make ends meet 331 (76.8) 


\section{Step 4: Dimensionality \& Recalibration}

The 35 Self-Care Decisions Scale items fit best into a 6-factor multidimensional structure in exploratory factor analysis (RMSEA $=0.05, \mathrm{CFI}=0.96, \mathrm{TLI}=0.94$, and $\mathrm{SRMR}=0.04)$.

Based on primary item loadings (Table 3 ) we identified six types of contextual factors that influence selfcare decisions about symptoms - there were no alternative factor loadings above a priori thresholds.

[Insert Table 3 about here]

Each represents a distinct and separately scored scale on the Self-Care Decisions Scale. Scales were labeled 'external,' 'urgency,' 'uncertainty,' 'cognitive/affective,' 'waiting/cue competition,' and 'concealment' based on the initial literature review and the content of the items that significantly loaded onto that scale (Table 4). Correlations between scales ranged from 0.35 (urgency and uncertainty) to 0.13 (urgency and concealment).

Table 4. Interpretations of the Six Scales of the Self-Care Decisions Scale

\begin{tabular}{|ll|}
\hline Scale & Interpretation \\
\hline Urgency & $\begin{array}{l}\text { The extent to which input from other people influences self-care decision } \\
\text { making. Higher scores indicate that self-care decision making is very } \\
\text { influenced by the input of others. }\end{array}$ \\
\hline Uncertainty & $\begin{array}{l}\text { The extent to which the perception of urgency or high stakes influences the } \\
\text { patient's self-care decision making. Higher scores indicate that the patient's } \\
\text { self-care decision making is very influenced by the perception that making a } \\
\text { decision about what to do about the symptom is urgent or important. }\end{array}$ \\
\hline $\begin{array}{l}\text { The extent to which uncertainty or ambiguity, from incomplete information } \\
\text { and/or difficulty interpreting the symptom, influence decision making. Higher } \\
\text { scores indicate that the patient's self-care decision making is very influenced } \\
\text { by being unsure about the cause or meaning of the symptom. }\end{array}$ \\
\hline $\begin{array}{l}\text { Waiting/Cue } \\
\text { Competition }\end{array}$ & $\begin{array}{l}\text { The extent to which the patient's thoughts or feelings influence decision } \\
\text { making. Higher scores indicate that that patient's thoughts and/or feelings } \\
\text { interfere with or prevent decision making. }\end{array}$ \\
\hline $\begin{array}{l}\text { The extent to which situational factors delay decision making. Higher scores } \\
\text { indicate that the patient is more likely to delay making a decision about their } \\
\text { self-care because of competing priorities and/or a perception that the decision } \\
\text { is not urgent. }\end{array}$ \\
\hline $\begin{array}{l}\text { The extent to which a desire to hide the symptom from others influences } \\
\text { decision making. Higher scores indicate that the patient's self-care decision } \\
\text { making is very influenced by a desire to conceal the symptom from others. }\end{array}$ \\
\hline
\end{tabular}

Scoring Note: Each scale is a separate standardized score that can range from 0-100. 
Four items were associated with the scale we labeled 'external.' Although all items were significant discriminators between low and high levels of external factors driving decision-making (Table 5), item 20 "someone else recognized the symptom before I did," had the lowest value for discrimination, and provided the least information about the influence of external factors (Figure 2). Further, based on category characteristic curves (Additional File 1), there had to be extremely high levels of the external influence (i.e. outside of the $95 \%$ confidence interval) for respondents to choose any response option above 1 (i.e. no influence). Therefore, item 20-was dropped from the 'external' scale.

Figure 2: Self-Care Decisions Scale Item Information Functions

Figure 2 Legend: Each pre-calibration item is show within the six scales of the Self-Care Decisions Scale. On the x-axis, theta represents the mean observed trait and the scale is standard errors around theta. On the $y$-axis, items providing more information about the trait with respect to greater discrimination have higher curves; items providing less information about the trait have lower curves, particularly those with a peak less than one. 


\section{Table 5: Scale-Specific Item Discrimination}

External

Discrimination within Scale

Others gave me advice

$2.238 \pm 0.284, p<0.001$

Others helped me to make a decision

$3.094 \pm 0.570, p<0.001$

Different people gave different advice about my symptom

$1.255 \pm 0.171, p<0.001$

Someone else recognized the symptom before I did

$1.134 \pm 0.180, p<0.001$

\section{Urgency}

I thought about decisions I made in the past when I had a similar symptom

The symptom got worse suddenly

$1.536 \pm 0.177, p<0.001$

When I had this symptom, I knew something was wrong

$1.434 \pm 0.168, p<0.001$

The symptom was severe or bothersome

$1.941 \pm 0.228, p<0.001$

I felt like something bad was going to happen

$1.700 \pm 0.199, p<0.001$

I felt I needed to make a decision quickly

$1.139 \pm 0.149, p<0.001$

\section{Uncertainty}

The symptom was different than what I expected

$1.492 \pm 0.150, p<0.001$

It wasn't clear to me what was causing the symptom

$1.574 \pm 0.159, p<0.001$

I didn't know what the symptom meant

$2.575 \pm 0.255, p<0.001$

I thought the symptom might be due to something else

$1.509 \pm 0.155, p<0.001$

I wasn't sure how important the symptom was

$1.621 \pm 0.161, p<0.001$

When I had the symptom, I didn't understand what was happening

$2.107 \pm 0.210, p<0.001$

The symptom was new to me

$2.505 \pm 0.278, p<0.001$

I recognized this symptom from the last time I had it

$-0.717 \pm 0.118, p<0.001$

The symptom was different than the last time I had it

$1.582 \pm 0.166, p<0.001$

\section{Cognitive/Affective}

I felt too sad to make a decision

$2.168 \pm 0.226, p<0.001$

My thinking was not clear so I could not make a decision

$2.352 \pm 0.230, p<0.001$

I felt too anxious to make a decision

$2.504 \pm 0.254, p<0.001$

I didn't feel well enough to make a decision 
I felt too tired to make a decision

$2.513 \pm 0.240, p<0.001$

I felt uncertain about what to do

$1.713 \pm 0.164, p<0.001$

Waiting/Cue Competition

I thought I could wait to make a decision

$1.400 \pm 0.153, p<0.001$

I felt that the symptom was nothing to worry about

$1.297 \pm 0.153, p<0.001$

The symptom changed slowly

$0.695 \pm 0.120, p<0.001$

I thought I could tolerate the symptom

$1.971 \pm 0.212, p<0.001$

Someone else needed my attention

$1.120 \pm 0.155, p<0.001$

I thought the symptom would go away on its own

$2.105 \pm 0.231, p<0.001$

Other things were more important at the time

$1.458 \pm 0.171, p<0.001$

\section{Concealment}

I felt embarrassed about my symptom

I didn't want to burden my family

I didn't want people to know about my symptom
$2.260 \pm 0.333, p<0.001$

$1.810 \pm 0.225, p<0.001$

$2.112 \pm 0.296, p<0.001$

Six items were associated with the scale we labeled 'urgency,' All items were significant discriminators between low and high levels of urgency (Table 5); but item 1, "I thought about decisions I made in the past when I had a similar symptom," had the lowest value for discrimination and not all response options discriminated significantly. In addition, item 1 provided almost no information about the influence of urgency (Figure 2), and there was a very low threshold for higher probability of respondents choosing higher response options. Therefore, item 1 was dropped from the 'urgency' scale.

Nine items loaded on the scale we labeled 'uncertainty.' All items were significant discriminators between low and high levels of uncertainty (Table 5); however, there were redundancies with respect to item information, especially involving these items: item 3 "The symptom was different than what I expected," and item 35 "The symptom was different than the last time I had it" (Figure 2). Additionally, item 33 "I recognized this symptom from the last time I had it" was the weakest discriminator and provided the least information about uncertainty. Items 3, 33 and 35 were omitted from the 'uncertainty' scale.

Six items were associated with the scale we labeled 'cognitive/affective.' All six items discriminated significantly (Table 5). However, for item 32 "I felt uncertain about what to do", not all response options were significant discriminators (Additional File 1) and item 32 also provided the least information about the influence of the individual's cognitive/affective state (Figure 2). Accordingly, item 32 was dropped from the 'cognitive/affective' scale. 
Seven items loaded on the scale we labeled 'waiting/cue competition.' All items discriminated significantly between low and high levels of waiting/cue competition (Table 5). However, items 25 "The symptom changed slowly" and 29 "Someone else needed my attention" had the lowest values for discrimination and provided the least information about the 'waiting/cue competition' scale (Figure 2). Hence, items 25 and 29 were omitted from the 'waiting/cue competition' scale.

Finally, three items loaded on the scale we labeled 'concealment.' All three items were significant discriminators between low and high levels of concealment (Table 5) and all items provided sufficient information about concealment (Figure 2). Accordingly, all three items were retained in the 'concealment' scale.

\section{Step 5: Construct Validity}

Correlations between the six new Self-Care Decisions Scale scales and the four domains of the Melbourne DMQ were tested (Table 6).

Table 6: Convergent Validity Testing with Melbourne Decision-Making Questionnaire Domains

External Urgency Uncertainty

Cognitive/ Affective
Waiting/ Cue

Competition

Concealment

\begin{tabular}{lllllll}
\hline Vigilance & - & - & - & - & - & - \\
\hline Buck Passing & 0.211 & - & - & 0.363 & 0.170 & 0.233 \\
\hline Procrastination & - & - & 0.178 & 0.402 & 0.239 & 0.266 \\
\hline Hypervigilance & 0.185 & - & 0.160 & 0.427 & - & 0.312
\end{tabular}

Values shown are significant $(p<0.05)$ linear correlations with Bonferroni correction applied

The Self-Care Decisions Scale external scale was modestly associated with buck passing and hypervigilance. The Self-Care Decisions Scale uncertainty scale was modestly associated with procrastination and hypervigilance. The Self-Care Decisions Scale cognitive/affective scale was associated with buck passing, procrastination, and hypervigilance. The Self-Care Decisions Scale waiting/cue competition scale was associated modestly with buck passing and procrastination. The SelfCare Decisions Scale concealment scale was associated with buck passing, procrastination and hypervigilance. No scale on the Self-Care Decisions Scale was significantly associated with the Melbourne DMQ vigilance domain, and the Self-Care Decisions Scale urgency scale was not associated with any Melbourne DMQ domain.

We also evaluated differences in scale scores of the Self-Care Decisions Scale between individuals with adequate and inadequate self-care management (Table 7). 


\begin{tabular}{|c|c|c|c|c|}
\hline & $\begin{array}{l}\text { Adequate Self-Care } \\
\text { Management } \\
\text { mean (sd) }\end{array}$ & $\begin{array}{l}\text { Inadequate } \\
\text { Self-Care } \\
\text { Management } \\
\text { mean (sd) }\end{array}$ & $\begin{array}{l}\mathrm{t}- \\
\text { statistic }\end{array}$ & $\begin{array}{l}\text { Effect } \\
\text { Size } \\
\text { Hedge's } \\
g\end{array}$ \\
\hline External & $31.56(26.89)$ & $23.76(22.35)$ & $\begin{array}{l}-2.97 \star \star \\
(p= \\
0.003)\end{array}$ & 0.32 \\
\hline Urgency & $65.14(20.54)$ & $55.40(24.02)$ & $\begin{array}{l}-4.34 * \star \\
(p< \\
0.001)\end{array}$ & 0.42 \\
\hline Uncertainty & $37.29(24.16)$ & $30.98(25.24)$ & $\begin{array}{l}-2.49 \star \star \\
(p= \\
0.013)\end{array}$ & 0.25 \\
\hline Cognitive/Affective & $25.54(25.42)$ & $26.22(26.52)$ & $\begin{array}{l}0.25 \\
(p=0.8)\end{array}$ & 0.03 \\
\hline $\begin{array}{l}\text { Waiting/Cue } \\
\text { Competition }\end{array}$ & $39.82(23.01)$ & $40.14(23.23)$ & $\begin{array}{l}0.13 \\
(p= \\
0.89)\end{array}$ & 0.01 \\
\hline Concealment & 36.75 (31.39) & 34.00 (28.53) & $\begin{array}{l}-0.88 \\
(p= \\
0.38)\end{array}$ & 0.09 \\
\hline
\end{tabular}

${ }^{\text {a }}$ Adequate self-care management is defined as a score $\geq 70$ on the SC-CII Management Scale ** p-value $<0.05$

There were significant differences in the scores on the external, urgency, and uncertainty scales. This partially supported our hypothesis that the scales of the Self-Care Decisions Scale would correlate with adequate self-care. Individuals with higher urgency had statistically significantly higher self-care management, as hypothesized. However, those with higher uncertainty also had higher self-care management scores.

\section{Step 6: Precision and Reliability}

Using IRT, test information function graphs along with plotted standard errors inform the range of underlying contextual factor where the scale is most precise; these data are provided in Figure 3. Using 
confirmatory factor analysis with recalibrated domains, multidimensional reliability (i.e., factor determinacy score) was high at 0.86 .

Figure 3: Recalibrated Test Information Functions for each scale of the Self-Care Decisions Scale

Figure 3 Legend: Each post-calibration scale of the Self-Care Decisions Scale is presented regarding the degree to which the factor items collectively inform the trait (left y-axis - information), and range of underlying trait ( $\mathrm{x}$-axis with theta representing the mean observed trait and the scale is standard errors around theta) where the scale is most precise (right $y$-axis - standard error).

\section{Scoring and Reference Ranges}

Separate standardized scoring (fixed score range from 0-100) is recommended for the six scales of the Self-Care Decisions Scale. There is no total score. Mean \pm standard deviation of standardized scores were external $(26.30 \pm 24.28)$, urgency $(58.57 \pm 25.38)$, uncertainty $(33.03 \pm 25.04)$, cognitive/affective (26.00 \pm 26.04$)$, waiting/cue competition $(40.04 \pm 23.13)$, and concealment $(34.89 \pm 29.48)$ in this derivation sample (Figure 4).

Figure 4: Standardized Scores on the Self-Care Decisions Scale

Figure 4 Legend: The mean and standard deviation of the standardized scores for each scale of the SelfCare Decisions Scale in the current sample are displayed. 
Table 3: Self-Care Decisions Scale Item Geomin Loadings and Multidimensional Structure

\begin{tabular}{|c|c|c|c|c|c|c|}
\hline $\begin{array}{l}\text { Self-Care } \\
\text { Decisions Scale } \\
\text { Item }\end{array}$ & External & Urgency & Uncertainty & $\begin{array}{l}\text { Cognitive/ } \\
\text { Affective }\end{array}$ & $\begin{array}{l}\text { Waiting/ } \\
\text { Cue } \\
\text { Competition }\end{array}$ & Concealment \\
\hline
\end{tabular}

1. I thought

0.535

about

decisions I

made in the

past when I

had a similar

symptom

1. Others gave

\subsection{9}

me advice

1. The

0.522

symptom

was different

than what I

expected

1. It wasn't

0.555

clear to me

what was

causing the

symptom

1. The

0.672

symptom got

worse

suddenly

1. I didn't know

0.739

what the

symptom

meant

1. When I had

0.611

this

symptom, I

knew

something

was wrong

1. I felt too sad

0.716

to make a

decision 
1. I thought the symptom

might be due

to something

else

1. Others

\subsection{4}

helped me to

make a

decision

1. I felt

embarrassed

about my

symptom

1. I didn't want

to burden my

family

1. Different

people gave

different

advice about

my symptom

1. I wasn't sure

how

important

the symptom

was

1. My thinking

\subsection{0}

was not clear

so I could

not make a

decision

1. I thought I

could wait to

make a

decision

1. When I had

the

symptom, I

didn't

understand

what was

happening 
1. The symptom

was severe

or

bothersome

1. I felt like

something

bad was

going to

happen

1. Someone

\subsection{3}

else

recognized

the symptom

before I did

1. I felt too

\subsection{1}

anxious to

make a

decision

1. The

\subsection{7}

symptom

was new to

me

1. I didn't want people to know about my symptom

1. I felt that the

symptom was nothing to worry about

1. The symptom changed slowly

1. I didn't feel well enough to make a decision

1. I thought I 
tolerate the

symptom

1. I felt I needed

0.407

to make a

decision

quickly

1. Someone else needed

my attention

1. I thought the

symptom

would go

away on its

own

1. I felt too tired

0.808

to make a

decision

1. I felt

0.555

uncertain

about what

to do

1. I recognized

$-0.711$

this

symptom

from the last

time I had it

1. Other things

0.506

were more

important at

the time

1. The

0.479

symptom

was different

than the last

time I had it

\section{Discussion}

The Self-Care Decisions Scale is a 27-item self-report instrument that measures contextual factors influencing self-care decisions about symptoms with six scales: 'external,' 'urgency,' 'uncertainty,' 
'cognitive/affective,' 'waiting/cue competition,' and 'concealment.' To our knowledge, this is the first instrument to operationalize naturalistic decision making to measure the contextual factors that influence self-care decisions. The instrument was developed with the input of clinicians, researchers, and adults with chronic illness, and content validity testing demonstrated that the retained items are relevant to and comprehensively cover the construct of naturalistic decision making as it relates to the self-care of symptoms. Further, comprehensibility of the instrument was confirmed by adults with chronic illness who completed cognitive interviews. The graded response IRT approach allowed us to eliminate items that did not discriminate well between varying levels of the underlying contextual factor. The psychometric properties of the instrument are promising with high multidimensional reliability (factor determinacy score $=0.86)$ and initial evidence of construct validity.

The six scales of the Self-Care Decisions Scale can help clinicians and researchers to understand how adults make self-care decisions in response to their symptoms of chronic illness. A core premise of naturalistic decision making is that decisions take place in real-world environments that are dynamically evolving (2). As such, decisions often must be made with incomplete information, and this can lead the decision-maker to feel uncertain. The 'uncertainty' scale assesses this aspect of naturalistic decision making by measuring the extent to which self-care decision making is influenced by ambiguity about what is causing the symptom or what the symptom means. Situational factors in a dynamically changing real-world context (e.g., a symptom suddenly worsens) can also contribute to the assessment of how urgently a decision must be made. The 'urgency' scale measures the extent to which the decisionmaker is influenced by the perception that decision-making about what to do in response to the symptom is time-sensitive. The 'waiting/cue competition' scale captures the influence of situational factors that can delay decision making because of competing priorities or the perception that the decision is not urgent. Riegel, Dickson (3) describe situation awareness as not only recognizing and interpreting the symptom, but also assessing the symptom's severity or importance. Further, situation awareness is necessary to think through treatment options (i.e., mental simulation) and decide what to do. These three scales (uncertainty, urgency, and waiting/cue competition) capture aspects of situational awareness and provide insight into how patients use information about their symptoms to make decisions. For example, a patient with a high uncertainty score may need support in learning how to assess the severity of their symptoms.

The involvement of multiple individuals (e.g., family, clinicians) may enhance or complicate the decisionmaking process. Individuals who score high on the 'external' scale are influenced strongly by the input of others. Further research on caregiver contributions to self-care will be helpful in determining if this input from others is helpful or detrimental to the patient's decision-making. In fact, prior studies have shown that indeed family caregivers have an important role in supporting patients performing self-care $(18,19)$, but how this support influences decision-making is unclear. Some adults with chronic illness may instead wish to hide their symptoms from others. The 'concealment' scale measures this concept by assessing the extent to which a desire to hide symptoms influences decision making. 
One's cognitive or affective state at the time when a symptom occurs can also influence decision making, a concept that is measured by the 'cognitive/ affective' scale. For example, Riegel, Dickson (3) found that in adults with heart failure, illness characteristics (e.g., sleepiness, depression) confounded symptom recognition and made decision making more tentative.

The initial draft of the instrument included several items related to prior experience, which is another core contextual factor of naturalistic decision making. For adults with heart failure, prior experience was valuable in improving the patient's ability to recognize and interpret symptoms (3). Interestingly, these items discriminated well between respondents at the extremes (i.e., prior experience having no influence or a lot of influence), but intermediate response options did not discriminate significantly, and the items were eliminated during recalibration. Respondents to our cognitive interviews universally endorsed that they used prior experience to inform their decisions. Therefore, we hypothesize that prior experience is foundational to decision-making about self-care. In other words, when a patient has experience with a symptom, they use information recalled from that experience to inform their decision about what to do in the current situation. A lack of prior experience is reflected in the 'uncertainty' scale.

We assessed convergent validity using the Melbourne DMQ, which measures a related concept of coping with decisional conflict. Our hypothesis of significant associations between the recalibrated scales of the Self-Care Decisions Scale and the Melbourne DMQ domains was partially supported. As decisional coping and the naturalistic decision making process are two related, but distinct, constructs we expected only modest correlations between the two instruments. The highest correlations were found between the Self-Care Decisions Scale cognitive/affective scale and coping styles of hypervigilance, procrastination, and buck passing. In other words, individuals who were highly influenced by thoughts or feelings (i.e., higher score on the cognitive/affective scale) had decisional coping styles that were more maladaptive. The Melbourne DMQ pattern of hypervigilance was modestly associated with the Self-Care Decisions Scale external and uncertainty scales, which could suggest that for some individuals the input of others and incomplete information leads to a chaotic coping pattern. The Self-Care Decisions Scale concealment scale, which correlated with the Melbourne DMQ patterns of hypervigilance, buck passing, and procrastination, could be seen as a coping response. The Self-Care Decisions Scale urgency scale was not associated with any coping patterns perhaps because the perception of urgency from experiencing a symptom that is severe or worsening leads to swift decision making rather than decisional conflict, which is measured by the Melbourne DMQ. Together, these modest associations among some scales suggest that some aspects of decision-making measured by the new Self-Care Decisions Scale are distinct from decisional conflict that is measured by the Melbourne DMQ. Investigators who are interested in the contextual factors derived from the naturalistic decision making framework and also want to understand how people cope with decisional conflict may want to use both instruments in future research.

We used the Self-Care of Chronic IIIness Inventory (SC-CII) to evaluate criterion validity, hypothesizing that the decision making factors most likely to be associated with adequate self-care management would be urgency and uncertainty. The influence of external factors, urgency, and uncertainty differed significantly 
between those with (SC-CII management scale score $\geq 70$ ) and without adequate self-care management. These results confirm findings from other studies that the perception of urgency and importance prompts adults to engage in self-care (20). Surprisingly, there was more uncertainty in those with adequate selfcare management compared with those in whom self-care management was inadequate (37.29 vs. 30.98 , respectively). This difference may be explained by considering that the self-care management scale measures responses to symptoms that include calling the provider for guidance. People may be more tempted to call the provider if they feel uncertain about what to do when they have a symptom. Finally, those with adequate self-care were more influenced by the input of others (external scale). This suggests that those with adequate self-care management are more likely to consult with others (e.g., family, clinicians) when making decisions about what to do when experiencing symptoms. As noted earlier, additional research into the contribution of caregivers to the patient's decision-making process is needed.

In this study, we operationalized the construct of naturalistic decision making to identify six scales that measure contextual factors that influence decisions about symptoms. To the best of our knowledge, naturalistic decision making has not been tested widely and other measures of naturalistic decision making were not located. Further research on this construct would be useful. Several of the contextual factors measured by this new instrument appear to be amenable to interventions aimed at improving decision-making about symptoms. Doing so may improve self-care.

Limitations include a convenience sample that was predominantly female, White, and residing in the United States. All data were cross-sectional, and our response rate was $38.2 \%$. Low response rates are a common concern in online-administered surveys (21). We are unable to assess if there were significant differences between those who chose to complete the survey and those who did not, which might have biased our sample. Further testing in more diverse populations is needed to ensure generalizability to all adults with symptomatic chronic illness. We did not evaluate test-retest reliability in this study, so the stability of the decision-making pattern(s) is unknown, even though, by its nature, naturalistic decision making might not be stable across time. Short-term stability should be tested in future research.

\section{Conclusion}

Initial psychometric analysis in this derivation sample yielded promising results. Validity testing of the Self-Care Decisions Scale suggests that this new instrument contributes in unique ways to our understanding of the processes that persons use to make decisions about their symptoms. While additional testing is indicated, the high multidimensional reliability and strong content validity confirmed by patients, researchers, and clinicians, suggests that the Self-Care Decisions Scale may be useful in future research. Further testing with other measures of decision making and in other populations will be useful in identifying the unique place of naturalistic decision-making in patient self-care behavior.

\section{Abbreviations}


CFI: Comparative Fit Index

COSMIN: COnsensus-based Standards for the selection of health Measurement Instruments

CVI: Content Validity Index

I-CVI: Item Content Validity Index

IRT: Item Response Theory

FIML: Full information maximum likelihood estimation

Melbourne DMQ: Melbourne Decision Making Questionnaire

RMSEA: root mean square error of approximation

SC-Cll: Self- Care of Chronic Illness Inventory

S-CVI: Scale Content Validity Index

SRMR: standardized root mean square residual

TLI: Tucker-Lewis index

\section{Declarations}

Ethics Approval and Consent to Participate: Ethical approval was obtained from the University of Pennsylvania Institutional Review Board (Protocol \#: 844892). Ethical approval included a waiver of written documentation of informed consent.

Consent for Publication: Not applicable

Availability of Data and Materials: The datasets used and/or analyzed during the current study are available from the corresponding author on reasonable request.

Competing Interests: The authors declare that they have no competing interests

Funding: Australian Catholic University (Grant \#: 060.0602.4.581955.xxxx.2406.0077)

Authors' contributions: All authors read and approved the final manuscript.

SP: Methodology, Formal Analysis, Investigation, Writing- Original Draft, Visualization

CL: Methodology, Formal Analysis, Data Curation, Writing - Original Draft, Visualization

SA: Methodology, Formal Analysis, Data Curation, Writing - Review \& Editing 
KF: Methodology, Formal Analysis, Writing- Review \& Editing

AS: Methodology, Formal Analysis, Writing - Review \& Editing

EV: Methodology, Formal Analysis, Writing - Review \& Editing

HW: Methodology, Formal Analysis, Writing - Review \& Editing

DW: Methodology, Writing - Review \& Editing

TJ: Conceptualization, Methodology, Formal Analysis, Writing - Review \& Editing, Funding Acquisition

BR: Conceptualization, Methodology, Formal Analysis, Writing- Original Draft, Supervision, Funding Acquisition

Acknowledgements: We thank Victoria Vaughan Dickson, PhD, RN, FAHA, FHFSA, FAAN for her early contributions to item development.

\section{References}

1. Riegel B, Jaarsma T, Strömberg A. A middle-range theory of self-care of chronic illness. ANS Adv Nurs Sci. 2012;35(3):194-204.

2. Zsambok CE, Klein G. Naturalistic Decision Making. Mahwah, New Jersey: Lawrence Erlbaum Associates; 1997.

3. Riegel B, Dickson VV, Topaz M. Qualitative analysis of naturalistic decision making in adults with chronic heart failure. Nurs Res. 2013;62(2):91-8.

4. Mokkink LB, Terwee CB, Patrick DL, Alonso J, Stratford PW, Knol DL, et al. The COSMIN study reached international consensus on taxonomy, terminology, and definitions of measurement properties for health-related patient-reported outcomes. J Clin Epidemiol. 2010;63(7):737-45.

5. Terwee CB, Prinsen CAC, Chiarotto A, Westerman MJ, Patrick DL, Alonso J, et al. COSMIN methodology for evaluating the content validity of patient-reported outcome measures: a Delphi study. Qual Life Res. 2018;27(5):1159-70.

6. Hasson F, Keeney S, McKenna H. Research guidelines for the Delphi survey technique. J Adv Nurs. 2000;32(4):1008-15.

7. Polit DF, Beck CT, Owen SV. Is the CVI an acceptable indicator of content validity? Appraisal and recommendations. Res Nurs Health. 2007;30(4):459-67.

8. Willis GB. Analysis of the Cognitive Interview in Questionnaire Design: Oxford University Press; 2015. 
9. Goodman RA, Posner SF, Huang ES, Parekh AK, Koh HK. Defining and measuring chronic conditions: imperatives for research, policy, program, and practice. Prev Chronic Dis. 2013;10:E66.

10. Howard MC. A Review of Exploratory Factor Analysis Decisions and Overview of Current Practices: What We Are Doing and How Can We Improve? International Journal of Human-Computer Interaction. 2016;32(1):51-62.

11. Hu Lt, Bentler PM. Cutoff criteria for fit indexes in covariance structure analysis: Conventional criteria versus new alternatives. Structural Equation Modeling: A Multidisciplinary Journal. 1999;6(1):155.

12. Schermelleh-Engel $\mathrm{K}$, Moosbrugger $\mathrm{H}$, Müller $\mathrm{H}$. Evaluating the Fit of Structural Equation Models: Tests of Significance and Descriptive Goodness-of-Fit Measures. Methods of Psychological Research. 2003;8(2):23-74.

13. Nguyen TH, Han HR, Kim MT, Chan KS. An introduction to item response theory for patientreported outcome measurement. Patient. 2014;7(1):23-35.

14. Mann L, Burnett P, Radford M, Ford S. The Melbourne Decision Making Questionnaire: An instrument for measuring patterns for coping with decisional conflict. Journal of Behavioral Decision Making. 1997;10(1):1-19.

15. Riegel B, Barbaranelli C, Sethares KA, Daus M, Moser DK, Miller JL, et al. Development and initial testing of the self-care of chronic illness inventory. J Adv Nurs. 2018;74(10):2465-76.

16. Riegel B, Lee CS, Dickson VV, Carlson B. An update on the self-care of heart failure index. J Cardiovasc Nurs. 2009;24(6):485-97.

17. Barbaranelli C, Lee CS, Vellone E, Riegel B. The problem with Cronbach's Alpha: comment on Sijtsma and van der Ark (2015). Nurs Res. 2015;64(2):140-5.

18. Vellone E, Biagioli V, Durante A, Buck HG, lovino P, Tomietto M, et al. The Influence of Caregiver Preparedness on Caregiver Contributions to Self-care in Heart Failure and the Mediating Role of Caregiver Confidence. J Cardiovasc Nurs. 2020;35(3):243-52.

19. Durante A, Paturzo M, Mottola A, Alvaro R, Vaughan Dickson V, Vellone E. Caregiver Contribution to Self-care in Patients With Heart Failure: A Qualitative Descriptive Study. J Cardiovasc Nurs. 2019;34(2):E28-e35.

20. Xu J, Gallo JJ, Wenzel J, Nolan MT, Budhathoki C, Abshire M, et al. Heart Failure Rehospitalization and Delayed Decision Making: The Impact of Self-care and Depression. J Cardiovasc Nurs. 2018;33(1):30-9. 
21. Arafa AE, Anzengruber F, Mostafa AM, Navarini AA. Perspectives of online surveys in dermatology. Journal of the European Academy of Dermatology and Venereology. 2019;33(3):511-20.

\section{Figures}

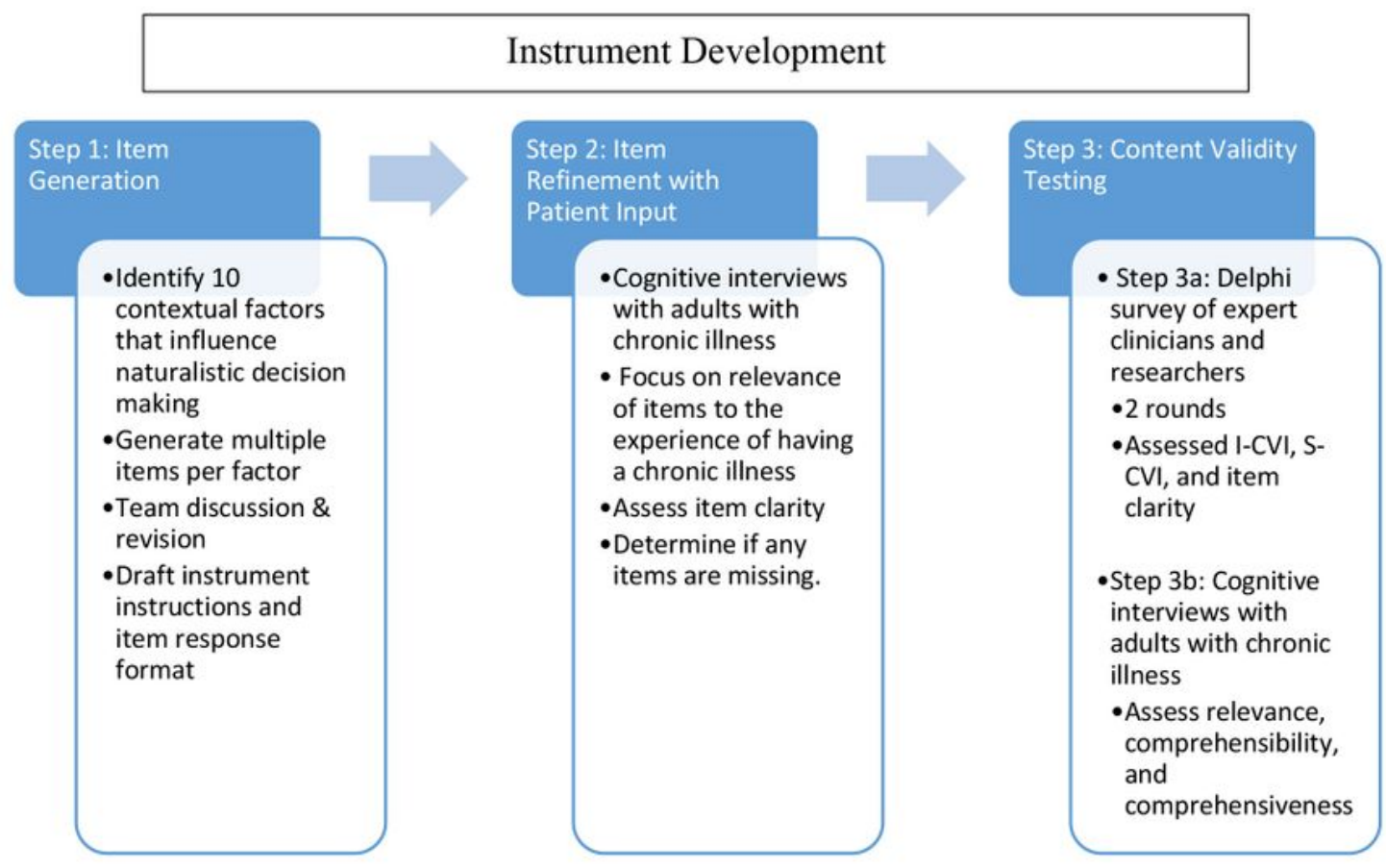

\section{Formal Psychometric Testing}

Step 4: Dimensionality
\& Recalibration
• Exploratory Factor
Analysis
• Graded response
item response
theory (IRT)-based
approaches
•Item discrimination
•item difficulty
•item and test
information curves
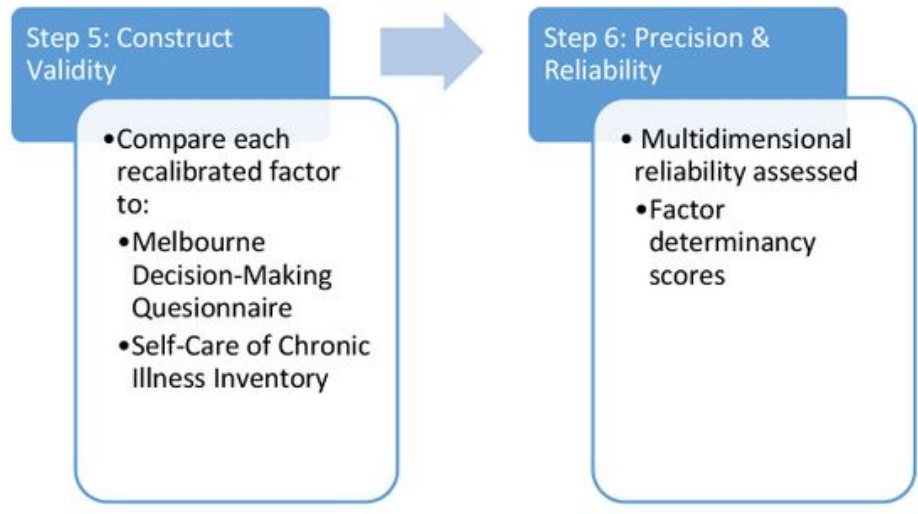

Figure 1 
Instrument Development and Formal Psychometric Testing Process.

External

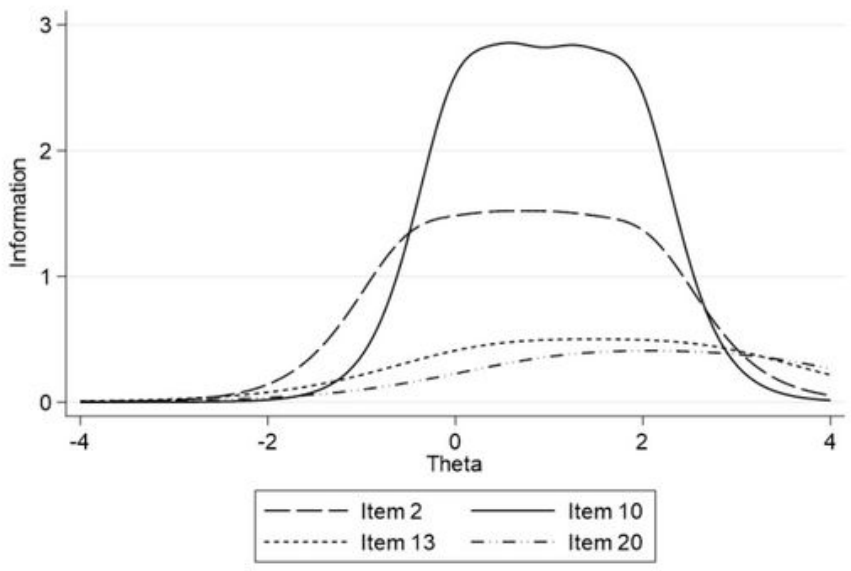

Uncertainty

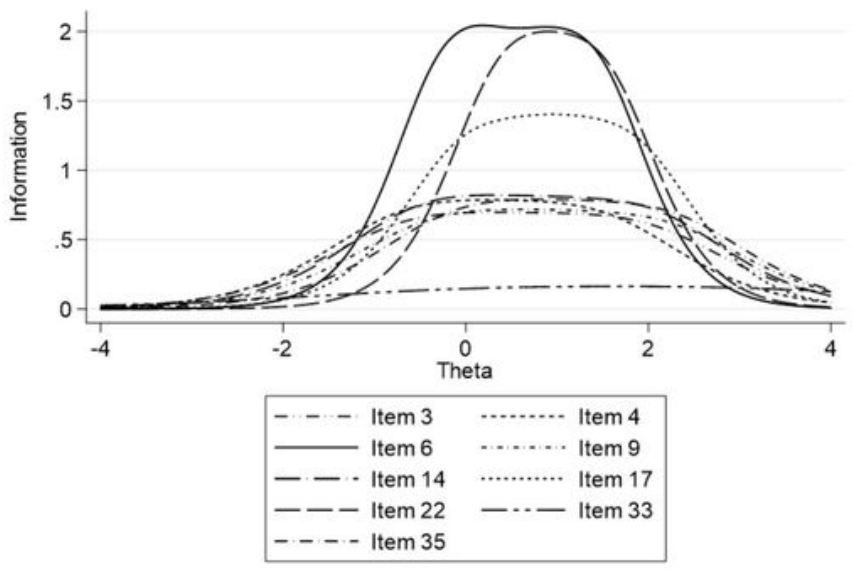

Waiting/Cue Competition

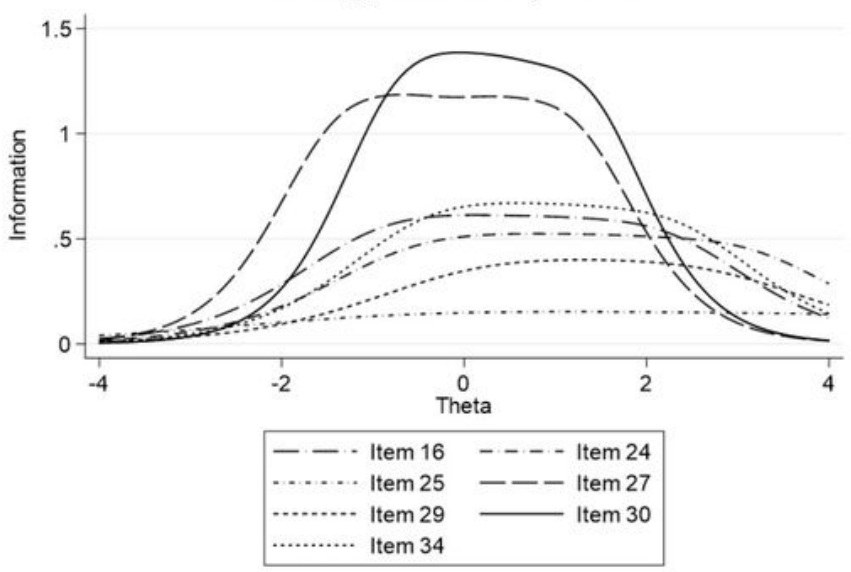

Urgency

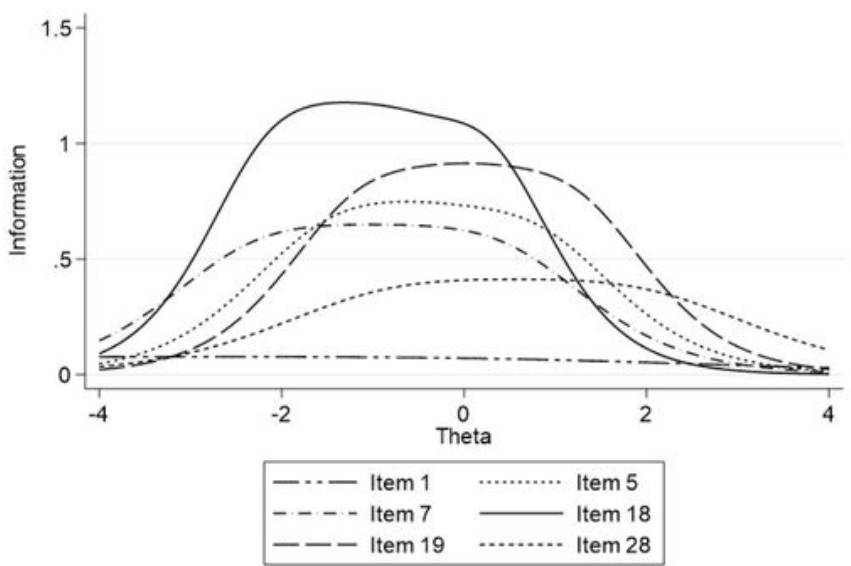

Cognitive/Affective

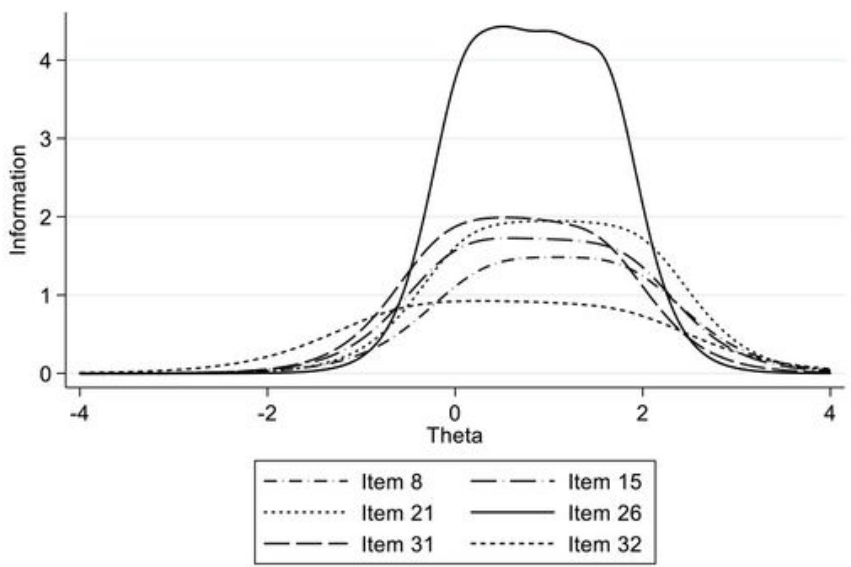

Concealment

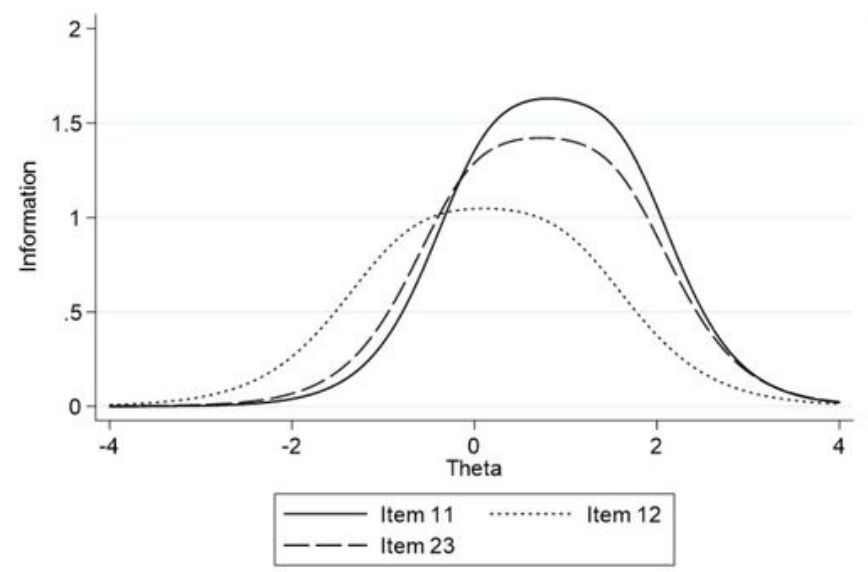

Figure 2

Self-Care Decisions Scale Item Information Functions. Each pre-calibration item is show within the six scales of the Self-Care Decisions Scale. On the x-axis, theta represents the mean observed trait and the scale is standard errors around theta. On the $y$-axis, items providing more information about the trait with 
respect to greater discrimination have higher curves; items providing less information about the trait have lower curves, particularly those with a peak less than one.

External

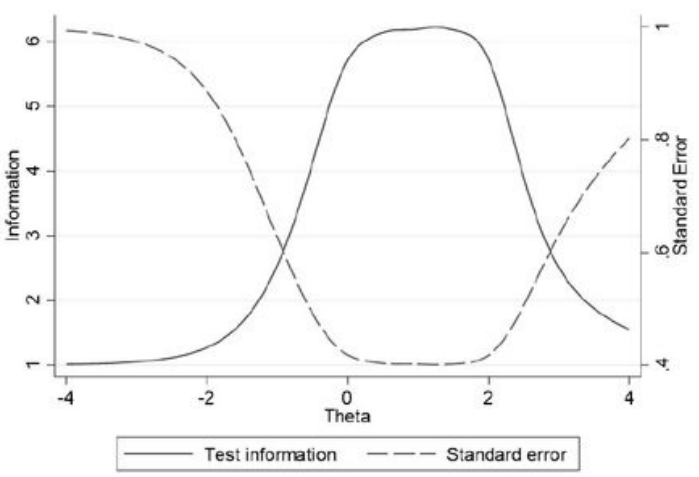

Uncertainty

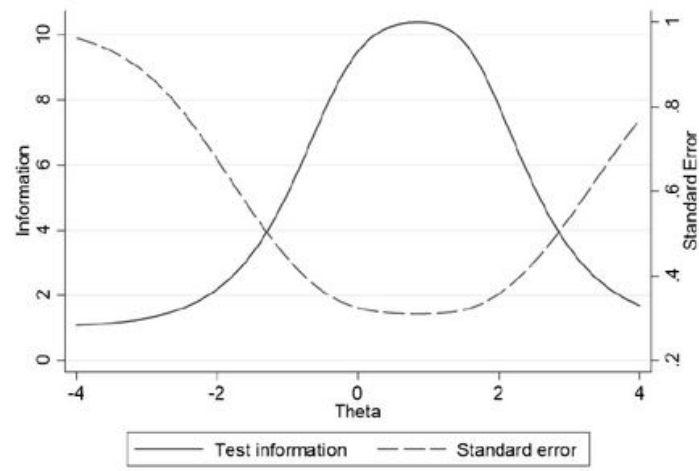

Waiting/Cue Competition

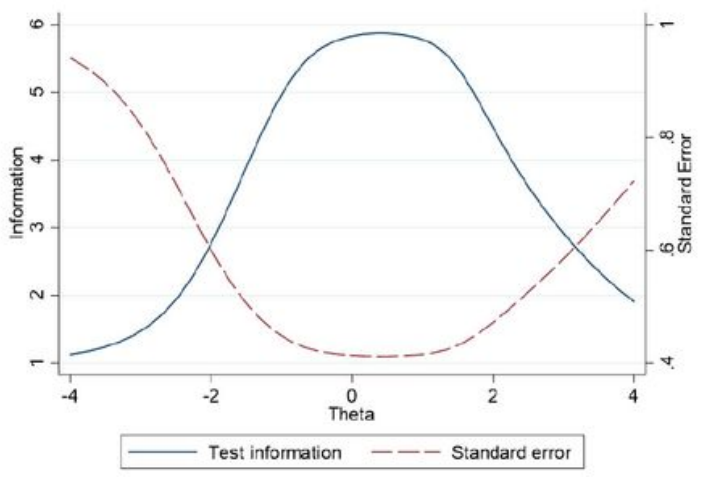

Urgency

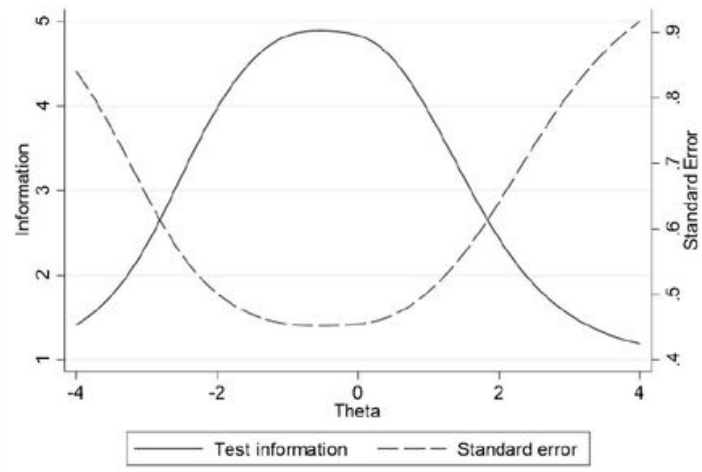

Cognitive/Affective

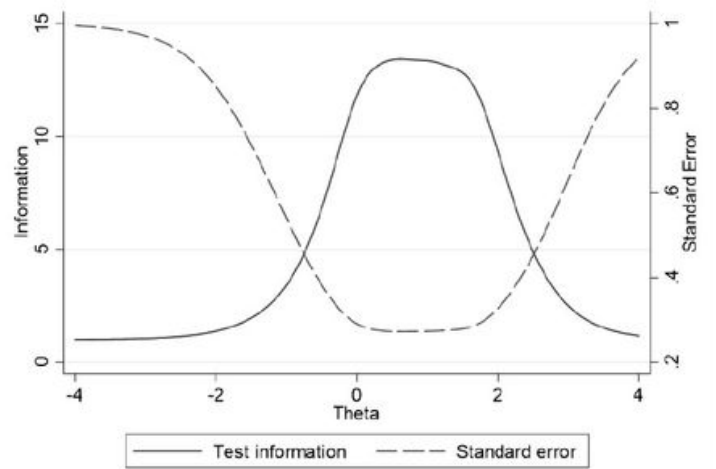

Concealment

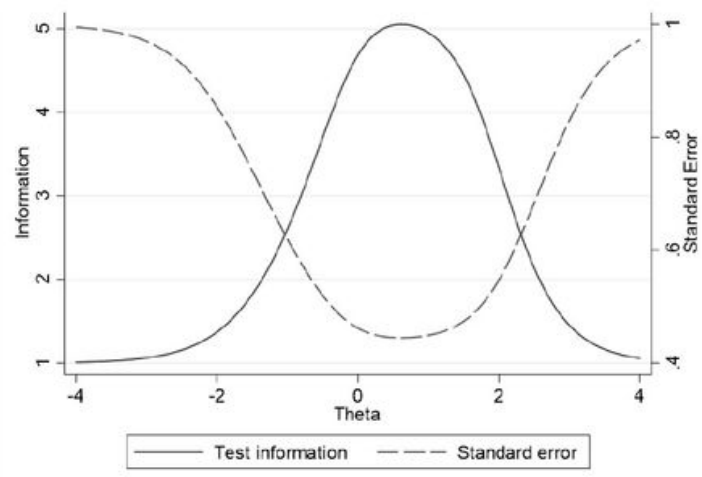

\section{Figure 3}

Recalibrated Test Information Functions for each scale of the Self-Care Decisions Scale. Each postcalibration scale of the Self-Care Decisions Scale is presented regarding the degree to which the factor items collectively inform the trait (left $y$-axis - information), and range of underlying trait ( $x$-axis with theta representing the mean observed trait and the scale is standard errors around theta) where the scale is most precise (right y-axis - standard error). 


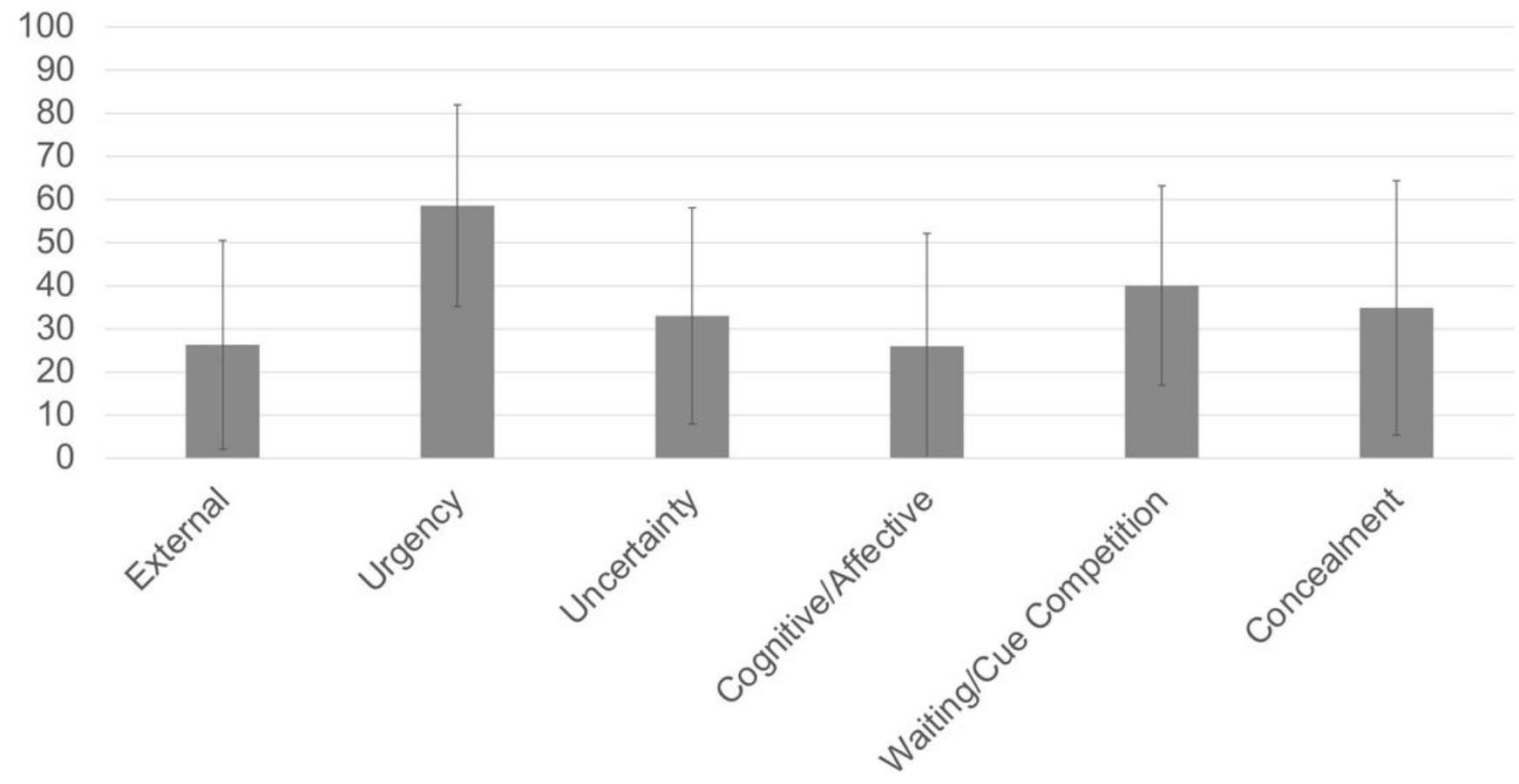

Figure 4

Standardized Scores on the Self-Care Decisions Scale. The mean and standard deviation of the standardized scores for each scale of the Self-Care Decisions Scale in the current sample are displayed.

\section{Supplementary Files}

This is a list of supplementary files associated with this preprint. Click to download.

- AdditionalFile1.pdf 